\title{
Global Attractors and Their Dimension Estimates for a Class of Generalized Kirchhoff Equations
}

\author{
Guoguang Lin, Lujiao Yang \\ Department of Mathematics, Yunnan University, Kunming, China \\ Email: gglin@ynu.edu.cn,1j112968y@163.com
}

How to cite this paper: Lin, G.G. and Yang, L.J. (2021) Global Attractors and Their Dimension Estimates for a Class of Generalized Kirchhoff Equations. Advances in Pure Mathematics, 11, 317-333. https://doi.org/10.4236/apm.2021.114020

Received: March 12, 2021

Accepted: April 22, 2021

Published: April 25, 2021

Copyright $\odot 2021$ by author(s) and Scientific Research Publishing Inc. This work is licensed under the Creative Commons Attribution International License (CC BY 4.0).

http://creativecommons.org/licenses/by/4.0/

\section{(c) (i) Open Access}

\begin{abstract}
In this paper, we studied the long-time properties of solutions of generalized Kirchhoff-type equation with strongly damped terms. Firstly, appropriate assumptions are made for the nonlinear source term $g(u)$ and Kirchhoff stress term $M(s)$ in the equation, and the existence and uniqueness of the solution are proved by using uniform prior estimates of time and Galerkin's finite element method. Then, abounded absorption set $B_{0 k}$ is obtained by prior estimation, and the Rellich-kondrachov's compact embedding theorem is used to prove that the solution semigroup $S(t)$ generated by the equation has a family of the global attractor $A_{k}$ in the phase space $E_{k}=H^{2 m+k} \times H^{k}$. Finally, linearize the equation and verify that the semigroups are Frechet diifferentiable on $E_{k}$. Then, the upper boundary estimation of the Hausdorff dimension and Fractal dimension of a family of the global attractor $A_{k}$ was obtained.
\end{abstract}

\section{Keywords}

Generalized Kirchhoff Equation, The Existence and Uniqueness of Solution, A Family of the Global Attractor, Dimension Estimation

\section{Introduction}

The objective of this paper is to study the following initial boundary value problem of the generalized Kirchhoff equation

$$
\begin{gathered}
u_{t t}+M\left(\left\|\nabla^{m} u\right\|_{p}^{p}\right)(-\Delta)^{2 m} u+\beta(-\Delta)^{2 m} u_{t}+g(u)=f(x) \\
u(x, t)=0, \frac{\partial^{i} u}{\partial v^{i}}=0, i=1,2, \cdots, 2 m-1, x \in \partial \Omega
\end{gathered}
$$




$$
u(x, 0)=u_{0}(x), u_{t}(x, 0)=u_{1}(x), x \in \Omega, t>0
$$

where $m>1, \quad p \geq 2, \Omega \subset R^{n}(n \geq 1)$ is a bounded domain with a smooth boundary $\partial \Omega, M(s) \in C^{2}\left([0,+\infty) ; R^{+}\right)$is a real function, $\beta(-\Delta)^{2 m} u_{t}(\beta>0)$ denotes strong damping term, $g(u)$ is nonlinear source term, $f(x)$ denotes the external force term. The assumption of $M(s)$ and $g(u)$ will be given later.

In 1883, German physicist G. Kirchhoff [1] first introduced the following model to study the free vibration of elastic strings

$$
\rho h \frac{\partial^{2} u}{\partial^{2} t}+\delta u_{t}=P_{0}+\frac{E h}{2 L}\left(\int_{0}^{L}\left|u_{x}\right|^{2} \mathrm{~d} x\right)+f(x), 0<x<L, t>0 .
$$

where the time variable is $t$, the elastic modulus is $E, h$ is the cross-sectional area, $L$ is the length of the string, $\rho$ is the mass density, $P_{0}$ is the initial axial tension, $\delta$ is the resistance coefficient, $f$ is the external force term, $u=u(x, t)$ is the lateral displacement at the space coordinate $x$ and the time $t$.

Since the 1980s, with the progress of science and technology and the continuous development of mathematical physics equations and Kirchhoff equation has been widely used, especially in measuring bridge vibration of engineering physics, so more and more scholars begin to pay close attention to and carries on the thorough study of Kirchhoff equation and a series of theories and research results in recent years, including the existence and uniqueness of the global solutions, global attractor and Hausdorff dimension and fractal dimension, the existence of random attractor, energy decay and blow-up of the solution, exponential attractor and inertial manifolds, etc. Among them, scholars have done a lot of research on the existence of global attractors for Kirchhoff-type equations with strong dissipation terms, the theoretical basis and research results can be found in the references ([2]-[9]).

In reference [10], Guoguang Lin, Yunlong Gao studied the existence and uniqueness of global solutions of a class of generalized Kirchhoff-type equations with nonlinear strong damping and their global attractors

$$
\begin{gathered}
u_{t t}+(-\Delta)^{m} u_{t}+\left(\alpha+\beta\left(\left\|D^{m} u\right\|^{2}\right)\right)(-\Delta)^{m} u+g(u)=f(x),(x, t) \in \Omega \times[0,+\infty) \\
u(x, t)=0, \frac{\partial^{i} u}{\partial v^{i}}=0, i=1,2, \cdots, m-1, x \in \partial \Omega, t \in[0,+\infty) \\
u(x, 0)=u_{0}(x), u_{t}(x, 0)=u_{1}(x), x \in \Omega
\end{gathered}
$$

By assuming the nonlinear source terms $g(u)$, the author verifies the appropriateness of the solution and proves the existence of the global attractor.

Recently, Lin Guoguang and Guan Liping [11] studied the global attractor of a high-order Kirchhoff-type equation with a strong nonlinear damping term and finite dimensional estimation of its Hausdorff dimension and Fractal dimension

$$
u_{t t}+M\left(\left\|D^{m} u\right\|^{2}\right)(-\Delta)^{m} u+\beta(-\Delta)^{m} u_{t}+\Delta g(u)=f(x)
$$

where $m>1, \Omega$ is a bounded domain with smooth homogeneous Dirichlet 
boundary $\partial \Omega \in R^{n}, f(x)$ denotes the external force, $\Delta g(u)$ denotes second order nonlinear source term, $M$ is a general function, $\beta>0, \beta(-\Delta)^{m} u_{t}$ is strong damping term. For more significant research results about the global attractor and its dimension estimation of Kirchhoff equation, please refer to the literature ([12] [13] [14] [15]).

In this paper, on the basis of literature [11], the rigid term $\left\|D^{m} u\right\|^{2}$ is extended to $\left\|D^{m} u\right\|_{p}^{p}$, the existence and uniqueness of global solutions of generalized nonlinear Kirchhoff-type equations are proved, and the existence of global attractors and their finite Hausdorff dimension and Fractal dimension of problems (1)-(3) are discussed.

For convenience, define the following spaces and notations

$$
\begin{aligned}
& H=L^{2}(\Omega), H_{0}^{m}(\Omega)=H^{m}(\Omega) \cap H_{0}^{1}(\Omega), H_{0}^{4 m}(\Omega)=H^{4 m}(\Omega) \cap H_{0}^{1}(\Omega), \\
& H_{0}^{2 m+k}(\Omega)=H^{2 m+k}(\Omega) \cap H_{0}^{1}(\Omega), E_{0}=H^{2 m}(\Omega) \times L^{2}(\Omega), f(x) \in L^{2}(\Omega), \\
& E_{k}=H_{0}^{2 m+k}(\Omega) \times H_{0}^{k}(\Omega),(k=1,2, \cdots, 2 m) .
\end{aligned}
$$

$(\cdot, \cdot)$ and $\|\cdot\|$ represent the inner product and norms of $H$ respectively, i.e.

$$
(u, v)=\int_{\Omega} u(x) v(x) \mathrm{d} x,(u, u)=\|u\|^{2},\|\cdot\|=\|u\|_{L^{2}(\Omega)},\|\cdot\|_{P}=\|u\|_{L^{P}(\Omega)},\|\cdot\|_{\infty}=\|u\|_{L^{\infty}(\Omega)} .
$$

Let's call $A_{k}(k=1,2, \cdots, 2 m)$ is the weak global attractor of $E_{0}$ to $E_{k}, B_{o k}$ is a bounded absorption set in $E_{k}, C_{i}>0(i=0,1,2, \cdots)$ is constant.

Assume that the nonlinear source term $g(u)$ in Equation (1.1) satisfies the following conditions

(A1) $g(u) \in C^{\infty}(R)$;

(A2) $J(u)=\int G(u) \mathrm{d} x$, where $G^{\prime}(u)=g(u) u_{t}$;

(A3) $J(u) \geq-\frac{\mu}{4}\left\|\nabla^{2 m+k} u\right\|^{2}-C$.

The Kirchhoff-type stress term satisfies the following conditions

(A4) $M(s) \in C^{2}\left([0,+\infty), R^{+}\right)$;

(A5) $\varepsilon+1=\mu_{0} \leq M(s) \leq \mu_{1}$, where $\mu=\left\{\begin{array}{l}\mu_{0}, \frac{\mathrm{d}}{\mathrm{d} t}\left\|\nabla^{m} u\right\|^{2} \geq 0 \\ \mu_{1}, \frac{\mathrm{d}}{\mathrm{d} t}\left\|\nabla^{m} u\right\|^{2}<0\end{array}\right.$

where $\mu, \mu_{0}, \mu_{1}$ are constant, and

$0<\varepsilon<\min \left\{\frac{\sqrt{1+2 \beta \lambda_{1}^{2 m}}-1}{2}, \frac{\mu_{0}+\sqrt{\mu_{0}^{2}-\lambda_{1}^{-2 m}}}{\lambda_{1}^{-2 m}}, \frac{\sqrt{1+\beta \lambda_{1}^{2 m}}-1}{2}, \frac{2 \mu_{0}}{\beta+\lambda_{1}^{-2 m}}\right\}, \quad \lambda_{1} \quad$ is the first eigenvalue of $-\Delta$ with homogeneous Dirichlet boundary conditions on $\Omega$.

\section{A Priori Estimate of Smooth Solution}

Lemma 1. Assume that the nonlinear terms $g(u), M(s)$ satisfies assumptions (A1)-(A5), and $f \in L^{2}(\Omega),\left(u_{0}, v_{0}\right) \in E_{0}=H^{2 m}(\Omega) \times L^{2}(\Omega)$, then the initial boundary value problem (1.1)-(1.3) has a smooth solution $(u, v) \in E_{0}$ and $v \in L^{2}\left(0, T ; H^{2 m}(\Omega)\right)$ 


$$
\|(u, v)\|_{E_{0}}^{2}=\left\|\nabla^{2 m} u\right\|^{2}+\|v\|^{2} \leq\|Y(0)\| \mathrm{e}^{-\alpha_{1} t}+\frac{C_{1}}{\alpha_{1}}\left(1-\mathrm{e}^{-\alpha_{1} t}\right) .
$$

where $v=u_{t}+\varepsilon u, \quad \alpha_{1}=\min \left\{a_{1}, \frac{a_{2}}{\mu}, 1\right\}, Y(0)=\left\|v_{0}\right\|^{2}+\mu\left\|\nabla^{2 m} u_{0}\right\|^{2}+2 J\left(u_{0}\right)$, so there's a non-negative real number $R_{0}=\sqrt{\frac{2 C_{1}}{\alpha_{1}}}$ and $t_{1}=\frac{1}{\alpha_{1}} \ln \left(\frac{\alpha_{1}\|Y(0)\|}{C_{1}}\right)$, and $\int_{0}^{T}\left\|\nabla^{2 m} v\right\|^{2} \mathrm{~d} t \leq C$, such that

$$
\|(u, v)\|_{E_{0}}^{2}=\left\|\nabla^{2 m} u\right\|^{2}+\|v\|^{2} \leq R_{0}^{2},\left(t>t_{1}\right) .
$$

Proof. Set $v=u_{t}+\varepsilon u$, take the inner product of both sides of Equation (1.1) with $v$ in $H$, we obtain

$$
\begin{aligned}
& \left(u_{t t}+M\left(\left\|\nabla^{m} u\right\|_{p}^{p}\right)(-\Delta)^{2 m} u+\beta(-\Delta)^{2 m} u_{t}+g(u), v\right)=(f(x), v) . \\
& \left(u_{t t}, v\right)=\left(v_{t}-\varepsilon v+\varepsilon^{2} u, v\right) \geq \frac{1}{2} \frac{\mathrm{d}}{\mathrm{d} t}\|v\|^{2}-\frac{2 \varepsilon+\varepsilon^{2}}{2}\|v\|^{2}-\frac{\varepsilon^{2}}{2} \lambda_{1}^{-2 m}\left\|\nabla^{2 m} u\right\|^{2} \text {. } \\
& \left(M\left(\left\|\nabla^{m} u\right\|_{p}^{p}\right)(-\Delta)^{2 m} u, v\right)=\left(M\left(\left\|\nabla^{m} u\right\|_{p}^{p}\right) \nabla^{2 m} u, \nabla^{2 m}\left(u_{t}+\varepsilon u\right)\right) \\
& =\frac{M\left(\left\|\nabla^{m} u\right\|_{p}^{p}\right)}{2} \frac{\mathrm{d}}{\mathrm{d} t}\left\|\nabla^{2 m} u\right\|^{2}+\varepsilon M\left(\left\|\nabla^{m} u\right\|_{p}^{p}\right)\left\|\nabla^{2 m} u\right\|^{2} \\
& \geq \frac{\mu}{2} \frac{\mathrm{d}}{\mathrm{d} t}\left\|\nabla^{2 m} u\right\|^{2}+\varepsilon \mu_{0}\left\|\nabla^{2 m} u\right\|^{2} \text {. }
\end{aligned}
$$

By using the Poincare's inequality, we obtain

$$
\begin{aligned}
\left(\beta(-\Delta)^{2 m} u_{t}, v\right) & =\beta\left\|\nabla^{2 m} v\right\|^{2}-\left(\beta \varepsilon(-\Delta)^{2 m} u, v\right) \\
& \geq \frac{\beta}{2}\left\|\nabla^{2 m} v\right\|^{2}+\frac{\beta \lambda_{1}^{2 m}}{2}\|v\|^{2}-\frac{1}{2}\left\|\nabla^{2 m} u\right\|^{2}-\frac{\beta^{2} \varepsilon^{2}}{2}\left\|\nabla^{2 m} v\right\|^{2} .
\end{aligned}
$$

The following estimation can be obtained from hypothesis (A2)

$$
\begin{aligned}
(g(u), v) & =\left(g(u), u_{t}\right)+\varepsilon(g(u), u)=\frac{\mathrm{d}}{\mathrm{d} t} \int G(u) \mathrm{d} x+\varepsilon(g(u), u) \\
& \geq \frac{\mathrm{d}}{\mathrm{d} t} \int G(u) \mathrm{d} x+\varepsilon^{2} \int G(u) \mathrm{d} x \geq \frac{\mathrm{d}}{\mathrm{d} t} J(u)+J(u) .
\end{aligned}
$$

By using the weighted Young's inequality, we obtain

$$
(f(x), v) \leq\|f(x)\| \cdot\|v\| \leq \frac{1}{2 \varepsilon^{2}}\|f(x)\|^{2}+\frac{\varepsilon^{2}}{2}\|v\|^{2} .
$$

Substitute inequality (2.4)-(2.8) into Equation (2.3), therefore

$$
\begin{aligned}
& \frac{\mathrm{d}}{\mathrm{d} t}\left[\|v\|^{2}+\mu\left\|\nabla^{2 m} u\right\|^{2}+2 J(u)\right]+\left(\beta \lambda_{1}^{2 m}-2 \varepsilon-2 \varepsilon^{2}\right)\|v\|^{2}+\left(\beta-\beta^{2} \varepsilon^{2}\right)\left\|\nabla^{2 m} v\right\|^{2} \\
& +\left(2 \varepsilon \mu_{0}-\varepsilon^{2} \lambda_{1}^{-2 m}-1\right)\left\|\nabla^{2 m} u\right\|^{2}+2 J(u) \leq \frac{\|f(x)\|^{2}}{\varepsilon^{2}}+C_{0} . \\
& \text { Let } a_{1}=\beta \lambda_{1}^{2 m}-2 \varepsilon-2 \varepsilon^{2} \geq 0, \quad a_{2}=2 \varepsilon \mu_{0}-\varepsilon^{2} \lambda_{1}^{-2 m}-1 \geq 0, \quad \beta-\beta^{2} \varepsilon^{2} \geq 0, \text { and }
\end{aligned}
$$


let $\alpha_{1}=\min \left\{a_{1}, \frac{a_{2}}{\mu}, 1\right\}, C_{1}=\frac{\|f(x)\|^{2}}{\varepsilon^{2}}+C_{0}$, then Equation (2.9) can be reduced to

$$
\frac{\mathrm{d}}{\mathrm{d} t} Y(t)+\alpha_{1} Y(t)+\left(\beta-\beta^{2} \varepsilon^{2}\right)\left\|\nabla^{2 m} v\right\|^{2} \leq C_{1} .
$$

According to hypothesis (A3)

$$
\left(\|v\|^{2}+\left\|\nabla^{2 m} u\right\|^{2}\right) \min \left(1, \frac{\mu}{2}\right) \leq\|v\|^{2}+\frac{\mu}{2}\left\|\nabla^{2 m} u\right\|^{2}+\left(\frac{\mu}{2}\left\|\nabla^{2 m} u\right\|^{2}+2 J(u)\right) \leq C .
$$

Then

$$
Y(t)=\|v\|^{2}+\mu\left\|\nabla^{2 m} u\right\|^{2}+2 J(u)>0
$$

By using the Gronwall's inequality, we get

$$
\|(u, v)\|_{E_{0}}^{2}=\left\|\nabla^{2 m} u\right\|^{2}+\|v\|^{2} \leq\|Y(0)\| \mathrm{e}^{-\alpha_{1} t}+\frac{C_{1}}{\alpha_{1}}\left(1-\mathrm{e}^{-\alpha_{1} t}\right) .
$$

And

$$
\varlimsup_{t \rightarrow \infty}\|(u, v)\|_{E_{0}}^{2} \leq \frac{C_{1}}{\alpha_{1}}
$$

So, there are constants $R_{0}=\sqrt{\frac{2 C_{1}}{\alpha_{1}}}$ and $t_{1}=\frac{1}{\alpha_{1}} \ln \left(\frac{\alpha_{1}\|Y(0)\|}{C_{1}}\right)>0$, we obtain

$$
\|(u, v)\|_{E_{0}}^{2}=\left\|\nabla^{2 m} u\right\|^{2}+\|v\|^{2} \leq R_{0}^{2},\left(t>t_{1}\right)
$$

The Lemma 1 is proved.

Lemma 2. Assume that the nonlinear terms $g(u), M(s)$ satisfies assumptions (A1)-(A5), and $f \in H^{k}(\Omega)$, $\left(u_{0}, v_{0}\right) \in E_{k}=H^{2 m+k}(\Omega) \times H^{k}(\Omega),(k=1,2, \cdots, 2 m)$, then the initial boundary value problem (1.1)-(1.3) has a smooth solution $u(x, t)$, $u_{t}(x, t)+\varepsilon u(x, t)=v(x, t)$ satisfy

$$
\|(u, v)\|_{E_{k}}^{2}=\left\|\nabla^{2 m+k} u\right\|^{2}+\left\|\nabla^{k} v\right\|^{2} \leq C_{2} \mathrm{e}^{-\alpha_{2} t}+\frac{C_{2}}{\alpha_{2}}\left(1-\mathrm{e}^{-\alpha_{2} t}\right) .
$$

where $\quad v=u_{t}+\varepsilon u, \quad \alpha_{2}=\min \left\{b_{1}, \frac{b_{2}}{\mu}\right\}, \quad Z(0)=\left\|\nabla^{k} v(0)\right\|^{2}+\mu\left\|\nabla^{2 m+k} u(0)\right\|^{2}$, so there are non-negative real number $R_{1}=\sqrt{\frac{2 C_{2}}{\alpha_{2}}}$ and $t_{2}=\frac{1}{\alpha_{2}} \ln \left(\frac{\alpha_{2}\|Z(0)\|}{C_{2}}\right)>0$, such that

$$
\|(u, v)\|_{E_{k}}^{2}=\left\|\nabla^{2 m+k} u\right\|^{2}+\left\|\nabla^{k} v\right\|^{2} \leq R_{1}^{2},\left(t>t_{2}\right) .
$$

Proof. Set $(-\Delta)^{k} v=(-\Delta)^{k} u_{t}+\varepsilon(-\Delta)^{k} u$, take the inner product of both sides of equation(1.1) with $(-\Delta)^{k} v$ in $H$, we obtain

$$
\left(u_{t t}+M\left(\left\|\nabla^{m} u\right\|_{p}^{p}\right)(-\Delta)^{2 m} u+\beta(-\Delta)^{2 m} u_{t}+g(u),(-\Delta)^{k} v\right)=\left(f(x),(-\Delta)^{k} v\right) \text {. }
$$




$$
\begin{aligned}
\left(u_{t t},(-\Delta)^{k} v\right) & =\left(v_{t}-\varepsilon v+\varepsilon^{2} u,(-\Delta)^{k} v\right) \\
& \geq \frac{1}{2} \frac{\mathrm{d}}{\mathrm{d} t}\left\|\nabla^{k} v\right\|^{2}-\frac{2 \varepsilon+\varepsilon^{2}}{2}\left\|\nabla^{k} v\right\|^{2}-\frac{\varepsilon^{2}}{2 \lambda_{1}^{2 m}}\left\|\nabla^{2 m+k} u\right\|^{2} .
\end{aligned}
$$

According to hypothesis (A5), and use a proof method similar to lemma 1, we can get

$$
\begin{aligned}
& \left(M\left(\left\|\nabla^{m} u\right\|_{p}^{p}\right)(-\Delta)^{2 m} u,(-\Delta)^{k} v\right) \\
& =M\left(\left\|\nabla^{m} u\right\|_{p}^{p}\right) \frac{1}{2} \frac{\mathrm{d}}{\mathrm{d} t}\left\|\nabla^{2 m+k} u\right\|^{2}+\varepsilon M\left(\left\|\nabla^{m} u\right\|_{p}^{p}\right)\left\|\nabla^{2 m+k} u\right\|^{2} \\
& \geq \frac{\mu}{2} \frac{\mathrm{d}}{\mathrm{d} t}\left\|\nabla^{2 m+k} u\right\|^{2}+\varepsilon \mu_{0}\left\|\nabla^{2 m+k} u\right\|^{2} .
\end{aligned}
$$

By using Poincare's inequality and Young's inequality, we have

$$
\begin{aligned}
& \left(\beta(-\Delta)^{2 m} u_{t},(-\Delta)^{k} v\right) \\
= & \beta\left((-\Delta)^{2 m} v-\varepsilon(-\Delta)^{2 m} u,(-\Delta)^{k} v\right) \\
\geq & \frac{\beta}{2}\left\|\nabla^{2 m+k} v\right\|^{2}+\frac{\beta \lambda_{1}^{2 m}}{2}\left\|\nabla^{k} v\right\|^{2}-\frac{1}{2}\left\|\nabla^{2 m+k} u\right\|^{2}-\frac{\beta^{2} \varepsilon^{2}}{2}\left\|\nabla^{2 m+k} v\right\|^{2} . \\
& \left(g(u),(-\Delta)^{k} v\right)=\|g(u)\|_{\infty}\left\|\nabla^{2 k} v\right\| \geq-\frac{\beta}{8}\left\|\nabla^{2 m+k} v\right\|^{2}-C_{3} .
\end{aligned}
$$

where $C_{3}=\frac{2\|g(u)\|_{\infty}}{\beta \lambda_{1}^{2 m-k}}$.

$$
\left(f(x),(-\Delta)^{k} v\right)=\left(\nabla^{k} f(x), \nabla^{k} v\right) \leq \frac{\left\|\nabla^{k} f\right\|^{2}}{2 \varepsilon^{2}}+\frac{\varepsilon^{2}}{2}\left\|\nabla^{k} v\right\|^{2} .
$$

Substitute inequality (2.19)-(2.23) to (2.18), therefore

$$
\begin{aligned}
& \frac{\mathrm{d}}{\mathrm{d} t}\left[\left\|\nabla^{k} v\right\|^{2}+\mu\left\|\nabla^{2 m+k} u\right\|^{2}\right]+\left(\beta \lambda_{1}^{2 m}-2 \varepsilon-2 \varepsilon^{2}\right)\left\|\nabla^{k} v\right\|^{2} \\
& +\left(2 \varepsilon \mu_{0}-\frac{\varepsilon^{2}}{\lambda_{1}^{2 m}}-1\right)\left\|\nabla^{2 m+k} u\right\|^{2}+\left(\frac{3 \beta}{4}-\beta^{2} \varepsilon^{2}\right)\left\|\nabla^{2 m+k} v\right\|^{2} \leq \frac{\left\|\nabla^{k} f(x)\right\|^{2}}{\varepsilon^{2}}+C_{3} .
\end{aligned}
$$

Obviously, there is a non-negative $\varepsilon$, such that $b_{1}=\beta \lambda_{1}^{2 m}-2 \varepsilon-2 \varepsilon^{2} \geq 0$, $b_{2}=2 \varepsilon \mu_{0}-\frac{\varepsilon^{2}}{\lambda_{1}^{2 m}}-1 \geq 0, \frac{3 \beta}{4}-\beta^{2} \varepsilon^{2} \geq 0$, let $\alpha_{2}=\min \left\{b_{1}, \frac{b_{2}}{\mu}\right\}$, $C_{2}=\frac{\left\|\nabla^{k} f(x)\right\|^{2}}{\varepsilon^{2}}+C_{3}$, then $(2.24)$ can reduce to

$$
\frac{\mathrm{d}}{\mathrm{d} t} Z(t)+\alpha_{2} Z(t)+\left(\frac{3 \beta}{4}-\beta^{2} \varepsilon^{2}\right)\left\|\nabla^{2 m+k} v\right\|^{2} \leq C_{2} .
$$

where

$$
Z(t)=\left\|\nabla^{k} v\right\|^{2}+\mu\left\|\nabla^{2 m+k} u\right\|^{2}>0
$$

By using Gronwall's inequality, we can get 


$$
\|(u, v)\|_{E_{k}}^{2}=\left\|\nabla^{k} v\right\|^{2}+\mu\left\|\nabla^{2 m+k} u\right\|^{2} \leq Z(0) \mathrm{e}^{-\alpha_{2} t}+\frac{C_{2}}{\alpha_{2}}\left(1-\mathrm{e}^{-\alpha_{2} t}\right) .
$$

and

$$
\varlimsup_{t \rightarrow \infty}\|(u, v)\|_{E_{k}}^{2} \leq \frac{C_{2}}{\alpha_{2}} .
$$

So, there are constants $R_{1}=\sqrt{\frac{2 C_{2}}{\alpha_{2}}}$ and $t_{2}=\frac{1}{\alpha_{2}} \ln \left(\frac{\alpha_{2}\|Z(0)\|}{C_{2}}\right)>0$, we obtain

$$
\|(u, v)\|_{E_{k}}^{2}=\left\|\nabla^{2 m+k} u\right\|^{2}+\left\|\nabla^{k} v\right\|^{2} \leq R_{1}^{2},\left(t>t_{2}\right) .
$$

The Lemma 2 is proved.

\section{Existence and Uniqueness of Solutions}

Theorem 1 Assume that the nonlinear terms $g(u), M(s)$ satisfies (A1)-(A5), $f \in H,\left(u_{0}, v_{0}\right) \in E_{0}$, then the initial boundary (1)-(3) exists a unique smooth solution $(u, v) \in L^{\infty}\left([0,+\infty) ; E_{0}\right)$. Proof. To prove the existence, the application of Calerkin's method is divided into the following three steps.

1) Approximate solution.

Suppose the eigenvector $\omega_{j}$ of $\left((-\Delta)^{2 m}\right) \omega_{j}=\lambda_{j}^{2 m} \omega_{j}$ generates an orthonormal basis for $H^{2 m}$, where $\lambda_{j}$ is the eigenvalue of $-\Delta$ with homogeneous Dirichlet boundary on $\Omega$, the k-order approximation $u_{k}(t)$ is defined as follows: $u_{k}(t) \in \operatorname{Span}\left[\omega_{1}, \omega_{2}, \cdots, \omega_{k}\right], u_{k}(t)=\sum_{j=1}^{k} g_{j k}(t) \omega_{j}$.

$$
\begin{aligned}
& \left(u_{k}^{\prime \prime}(t), \omega_{j}\right)+M\left(\left\|\nabla^{m} u_{k}(t)\right\|_{p}^{p}\right)\left(\nabla^{2 m} u_{k}(t), \nabla^{2 m} \omega_{j}\right) \\
& +\beta\left(\nabla^{2 m} u^{\prime}(t), \nabla^{2 m} \omega_{j}\right)+\left(g\left(u_{k}\right), \omega_{j}\right)=\left(f(x), \omega_{j}\right)
\end{aligned}
$$

where $j=1,2, \cdots, k$.

$$
u_{k}(0)=u_{0 k}, \quad u_{k}^{\prime}(0)=u_{1 k} \text { and } u_{0 k}, u_{1 k} \in \operatorname{Span}\left[\omega_{1}, \omega_{2}, \cdots, \omega_{k}\right] .
$$

In $H, u_{0 k} \rightarrow u_{0}, u_{1 k} \rightarrow v_{0}$, the system of ordinary differential equations with respect to $g_{j k}(t)$ can be determined on the interval $\left[0, t_{k}\right]$, we need to prove that $t_{k}=T$.

2) Prior estimate.

According to the conclusion and proof method of lemma $1,\left(u_{k}(t), u_{k}^{\prime}(t)\right)$ is uniformly bounded on $E_{0}$, that is

$$
\begin{gathered}
\left\|u_{k}(t)\right\| \leq R \\
\left\|\nabla^{2 m} u_{k}(t)\right\| \leq R \\
\left\|u_{k}^{\prime}(t)\right\| \leq R
\end{gathered}
$$

where $R$ is a constant independent of $k$. According to lemma2, we get $u_{k}^{\prime}(t) \in L^{2}\left(0, T ; L^{2}(\Omega)\right)$, therefore $t_{k}=T$, inequality (3.2)-(3.4) indicate $u_{k}(t)$ is bounded in $L^{\infty}\left(0, T ; H^{2 m}(\Omega)\right), u_{k}^{\prime}(t)$ is bounded in $L^{\infty}\left(0, T ; L^{2}(\Omega)\right)$, in fact, we can get $u_{k}(t) \in L^{\infty}\left(0,+\infty ; H^{2 m}(\Omega)\right)$ and $u_{k}^{\prime}(t) \in L^{\infty}\left(0,+\infty ; L^{2}(\Omega)\right)$. 
3) Limit process.

According to Danford-Pttes throrem, space $L^{2}\left(0, T ; H^{2 m}(\Omega)\right)$ is conjugated to space $L^{2}\left(0, T ; H^{-2 m}(\Omega)\right)$, space $L^{\infty}\left(0, T ; L^{2}(\Omega)\right)$ is conjugated to space $L^{\prime}\left(0, T ; L^{2}(\Omega)\right)$, select the subsequence $u_{k}^{*}(t)$ from the sequence $u_{k}(t)$, such that

$u_{k}^{*} \rightarrow u$ is weak * convergence in $L^{\infty}\left(0, T ; H^{2 m}(\Omega)\right)$;

$u_{k}^{* \prime} \rightarrow u^{\prime}$ is weak * convergence in $L^{\infty}\left(0, T ; L^{2}(\Omega)\right)$;

$u_{k}^{* \prime} \rightarrow u^{\prime}$ is weak convergence in $L^{2}\left(0, T ; H^{2 m}(\Omega)\right)$.

By the Rellich-Kohdrachov compact embedding theorem, $H^{2 m}(\Omega)$ is compact embedded in $L^{p}(\Omega)$ and $L^{2}(\Omega)$, then $\left(u_{k}(t), u_{k}^{* \prime}\right) \rightarrow\left(u, u^{\prime}\right)$ converges strongly almost everywhere in $L^{2}(0, T)$ and $L^{2}(0, T)$.

$$
\begin{aligned}
& M\left(\left\|u_{k}(t)\right\|_{p}^{p}\right) \rightarrow M\left(\|u(t)\|_{p}^{p}\right) \text { converges in } R^{+} ; \\
& M\left(\left\|u_{k}(t)\right\|_{p}^{p}\right)\left(\nabla^{2 m} u_{k}(t), \nabla^{2 m} \omega_{j}(t)\right) \rightarrow M\left(\|u(t)\|_{p}^{p}\right)\left(\nabla^{2 m} u, \nabla^{2 m} \omega_{j}\right) \text { is weak * }
\end{aligned}
$$

convergence in $L^{\infty}(0, T)$;

$\beta\left(\nabla^{2 m} u_{k}^{\prime}(t), \nabla^{2 m} \omega_{j}(t)\right) \rightarrow \beta\left(\nabla^{2 m} u^{\prime}(t), \nabla^{2 m} \omega_{j}\right)$ is weak * convergence in $L^{\infty}(0, T)$.

Because of $g\left(u_{k}\right) \in L^{\infty}\left(H_{0}^{k}\right)$, so

$\left(g\left(u_{k}\right), \omega_{j}\right) \rightarrow\left(g(u), \omega_{j}\right)$ is weak * convergence in $L^{\infty}(0, T)$.

$\left(u_{k}^{\prime \prime}(t), \omega_{j}\right)=\frac{\mathrm{d}}{\mathrm{d} t}\left(u_{k}^{\prime}(t), \omega_{j}\right) \rightarrow\left(u^{\prime \prime}, \omega_{j}\right)$ is converges in $D^{\prime}(0, T)$.

From Equation (3.1), the following formula can be derived

$\left(u^{\prime \prime}, \omega_{j}\right)+M\left(\left\|\nabla^{m} u\right\|_{p}^{p}\right)\left(\nabla^{2 m} u, \nabla^{2 m} \omega_{j}\right)+\beta\left(\nabla^{2 m} u^{\prime}(t), \nabla^{2 m} \omega_{j}\right)$ this is true for $\forall j$.

$+\left(g(u), \omega_{j}\right)=\left(f(x), \omega_{j}\right)$

By the density of the base $\omega_{1}, \omega_{2}, \cdots, \omega_{k}, \cdots$, then for $\forall \varphi \in H^{2 m}(\Omega)$, the following equation is established

$$
\left(u^{\prime \prime}, \varphi\right)+M\left(\left\|\nabla^{m} u\right\|_{p}^{p}\right)\left(\nabla^{2 m} u, \nabla^{2 m} \varphi\right)+\beta\left(\nabla^{2 m} u^{\prime}(t), \nabla^{2 m} \varphi\right)+(g(u), \varphi)=(f(x), \varphi)
$$

Then $u_{k}(0) \rightarrow u_{0}$ is weak convergence in $L^{2}(\Omega)$, and in $H^{2 m}(\Omega)$, we establish

$$
\begin{gathered}
u_{k}(0) \rightarrow u_{0} ; \\
\left.\left(u_{k}^{\prime}(0), \omega_{j}\right) \rightarrow\left(u^{\prime}, \omega_{j}\right)\right|_{t=0}=\left(u^{\prime}(0), \omega_{j}\right) ; \\
\left(u_{k}^{\prime}(0), \omega_{j}\right) \rightarrow\left(v_{0}, \omega_{j}\right)
\end{gathered}
$$

So $\left(u^{\prime}(0), \omega_{j}\right)=\left(v_{0}, \omega_{j}\right)$ is satisfied for all $\mathrm{j}$, so that existence can be proved.

Then prove the uniqueness of the solution.

Assume $u_{1}$ and $u_{2}$ are solutions of Equation (1.1), let $w=u_{1}-u_{2}$, and substitute $u_{1}$ and $u_{2}$ into this equation, we can obtain

$$
\begin{aligned}
& w_{t t}+M\left(\left\|\nabla^{m} u_{1}\right\|_{p}^{p}\right)(-\Delta)^{2 m} u_{1}-M\left(\left\|\nabla^{m} u_{2}\right\|_{p}^{p}\right)(-\Delta)^{2 m} u_{2} \\
& +\beta(-\Delta)^{2 m} w_{t}+g\left(u_{1}\right)-g\left(u_{2}\right)=0 .
\end{aligned}
$$


Take the inner product of both sides of Equation (3.5) with $w_{t}$ in $H$, then

$\frac{1}{2} \frac{\mathrm{d}}{\mathrm{d} t}\left\|w_{t}\right\|^{2}+\beta\left\|\Delta^{m} w_{t}\right\|^{2}+\left(M\left(\left\|\nabla^{m} u_{1}\right\|_{p}^{p}\right)(-\Delta)^{2 m} u_{1}-M\left(\left\|\nabla^{m} u_{2}\right\|_{p}^{p}\right)(-\nabla)^{2 m} u_{2}, w_{t}\right)$

$+\left(g\left(u_{1}\right)-g\left(u_{2}\right), w_{t}\right)=0$.

According to Sobolev embedding theorem, $H_{0}^{2 m}(\Omega) \subset L^{P}(\Omega)$, there exists constant $r>0$, we have

$$
\left\|\nabla^{m} u_{1}\right\|_{L^{p}(\Omega)}^{p} \leq r\left\|(-\Delta)^{m} u_{1}\right\|^{\frac{(2 m+n) p-2 n}{4 m}}\left\|u_{1}\right\|^{\frac{(2 m-n) p+2 n}{4 m}} .
$$

where

$$
\left\{\begin{array}{l}
\frac{2 n}{n+2 m} \leq p \leq \frac{2 n}{n-2 m}, n>2 m ; \\
\frac{2 n}{n+2 m} \leq p \leq \infty, n \leq 2 m .
\end{array}\right.
$$

By lemma 1, lemma 2, differential mean value theorem and Young's inequality, we can obtain

$$
\begin{aligned}
& \left(M\left(\left\|\nabla^{m} u_{1}\right\|_{p}^{p}\right)(-\Delta)^{2 m} u_{1}-M\left(\left\|\nabla^{m} u_{2}\right\|_{p}^{p}\right)(-\Delta)^{2 m} u_{2}, w_{t}\right) \\
& =\left(M\left(\left\|\nabla^{m} u_{1}\right\|_{p}^{p}\right)(-\Delta)^{2 m} u_{1}-M\left(\left\|\nabla^{m} u_{1}\right\|_{p}^{p}\right)(-\Delta)^{2 m} u_{2}\right. \\
& \left.\quad+M\left(\left\|\nabla^{m} u_{1}\right\|_{p}^{p}\right)(-\Delta)^{2 m} u_{2}-M\left(\left\|\nabla^{m} u_{2}\right\|_{p}^{p}\right)(-\Delta)^{2 m} u_{2}, w_{t}\right) \\
& \geq \frac{1}{2} M\left(\left\|\nabla^{m} u_{1}\right\|_{p}^{p}\right) \frac{\mathrm{d}}{\mathrm{d} t}\left\|\Delta^{m} w\right\|^{2}-\left(M^{\prime}(\xi)\left(\left\|\nabla^{m} u_{1}\right\|_{p}^{p}-\left\|\nabla^{m} u_{2}\right\|_{p}^{p}\right)(-\Delta)^{2 m} u_{2}, \omega_{t}\right) \\
& \geq \frac{\mu}{2} \frac{\mathrm{d}}{\mathrm{d} t}\left\|\Delta^{m} w\right\|^{2}-\left\|M^{\prime}(\xi)\right\| \cdot\left\|\nabla^{2 m} u_{2}\right\| \cdot\left\|\nabla^{2 m} \omega_{t}\right\| \cdot\left\|\nabla^{m} \omega\right\|_{\infty} \cdot C_{4} \cdot\left\|\nabla^{m} u_{1}\right\|_{L^{p-1}}^{p-1} \cdot\left\|\nabla^{m} u_{2}\right\|_{L^{p-1}}^{p-1} \\
& \geq \frac{\mu}{2} \frac{\mathrm{d}}{\mathrm{d} t}\left\|\Delta^{m} w\right\|^{2}-\frac{\beta}{2}\left\|\Delta^{m} w\right\|^{2}-\frac{C_{4}^{2}}{2 \beta}\left\|\Delta^{m} w_{t}\right\|^{2} . \\
& \quad \text { where } \xi=\theta u_{1}+(1-\theta) u_{2}, 0<\theta<1 .
\end{aligned}
$$

According to the hypothesis (A1), we get

$$
\begin{aligned}
\left(g\left(u_{1}\right)-g\left(u_{2}\right), w_{t}\right) & \geq-\left\|g^{\prime}(\xi)\right\|\left\|_{\infty}\right\| w\|\| w_{t}\left\|\geq-C_{5} C_{6}\right\| \nabla^{m} w\|\| w_{t} \| \\
& \geq-\frac{C_{5} C_{6}}{2}\left\|w_{t}\right\|^{2}-\frac{C_{5} C_{6}}{2}\left\|\nabla^{m} w\right\|^{2} \\
& \geq-\frac{C_{5} C_{6}}{2}\left\|w_{t}\right\|^{2}-\frac{C_{5} C_{6}}{2} \lambda_{1}^{-m}\left\|\Delta^{m} w\right\|^{2} .
\end{aligned}
$$

Substitute inequality (3.9), (3.10) to (3.6), we get

$$
\begin{aligned}
& \frac{\mathrm{d}}{\mathrm{d} t}\left(\left\|w_{t}\right\|^{2}+\mu\left\|\Delta^{m} w\right\|^{2}\right)+\left(2 \beta-\frac{C_{4}^{2}}{\beta}\right)\left\|\Delta^{m} w_{t}\right\|^{2} \\
& -\left(\beta+C_{5} C_{6} \lambda_{1}^{-m}\right)\left\|\Delta^{m} w\right\|^{2}-C_{5} C_{6}\left\|w_{t}\right\|^{2} \leq 0
\end{aligned}
$$

By using Gronwall's inequality, we get

$$
\frac{\mathrm{d}}{\mathrm{d} t}\left(\left\|w_{t}\right\|^{2}+\mu\left\|\Delta^{m} w\right\|^{2}\right) \leq C_{7}\left(\left\|w_{t}\right\|^{2}+\mu\left\|\Delta^{m} w\right\|^{2}\right)
$$


where $C_{7}=\max \left\{\frac{\beta+C_{5} C_{6} \lambda_{1}^{-m}}{\mu}, C_{5} C_{6}\right\}$.

So we can get

$$
\left(\left\|w_{t}(t)\right\|^{2}+\mu\left\|\Delta^{m} w(t)\right\|^{2}\right) \leq\left(\left\|w_{t}(0)\right\|^{2}+\mu\left\|\Delta^{m} w(0)\right\|^{2}\right) \mathrm{e}^{C_{7} t}=0 .
$$

Then $w_{t}(t)=0, \Delta^{m} w(t)=0$, so $w(t)=0, u_{1}=u_{2}$, then the uniqueness of solutions is proved.

The theorem 1 is proved completely.

\section{The Existence of the Family of Global Attractor}

Theorem 2 [16] Assume that $E$ is a Banach space, $\{S(t)\}_{t \geq 0}$ is a semigroup operator on $E$, and $S(t): E \rightarrow E, S(t+s)=S(t) S(s)(\forall t, s \geq 0), S(0)=I$, where $I$ is unit operator, suppose $S(t)$ satisfies the following conditions:

1) Semigroup $S(t)$ is uniformly bounded in E, that is for all $r>0$, it exists a constant $C(r)$, such that $\|u\|_{E} \leq r$,

$$
\|S(t) u\|_{E} \leq C(r)(\forall t \in[0, \infty)) ;
$$

2) It exists a bounded absorbing set $B_{0} \subset E$, that is for all $B \subset E$, it exists a constant $t_{0}$,

$$
S(t) B \subset B_{0}\left(t \geq t_{0}\right) ;
$$

3) $S(t)(t \geq 0)$ is completely continuous operator.

Thus there is a compact global attractor $A_{0}$ for the semigroup operator $S(t)$.

If the Banach space $E$ is changed to Hilbert space $E_{k}$ in theorem 2, the existence theorem of the family of the global attractors can be obtained.

Theorem 3 Under the assumption of lemma 1, lemma 2 and theorem 1, problem (1.1)-(1.3) exist a family of the global attractors $A_{k}$

$$
A_{k}=\omega\left(B_{0 k}\right)=\bigcap_{s \geq 0} \overline{t \geq s} S(t) B_{0 k} .
$$

where $B_{0 k}=\left\{(u, v) \in E_{k},\|(u, v)\|_{E_{k}}^{2}=\|u\|_{H_{0}^{2 m+k}(\Omega)}^{2}+\|v\|_{H_{0}^{K}(\Omega)}^{2} \leq C\right\}, \quad B_{0 k}$ is a bounded absorbing set in $E_{k}$, that is exists a compact set $A_{k} \subset E_{k} \subset E_{0}$ satisfies the following conditions:

1) $S(t) A_{k}=A_{k}(\forall t>0)$

2) $\lim _{t \rightarrow \infty} \operatorname{dist}\left(S(t) B, A_{k}\right)=0,\left(\forall B \subset E_{k}(\Omega)\right.$ is bounded set), where

$$
\lim _{t \rightarrow \infty} \operatorname{dist}\left(S(t) B, A_{k}\right)=\sup _{x \in B}\left(\inf _{y \in A_{k}}\|S(t) x-y\|_{E_{k}}\right) \rightarrow 0, t \rightarrow \infty .
$$

$S(t)$ is the solution semigroup generated by problem (1.1)-(1.3).

Proof. We need to prove the three conditions of theorem 2, according to theorem 1, lemma 2, we know the problem (1.1)-(1.3) could generate the solution semigroup $S(t), S(t): E_{k} \rightarrow E_{k}$.

1) According to lemma 1 and lemma 2, we know that for any bounded set $B \subset E_{k}$, and 


$$
\begin{gathered}
\left\{\|(u, v)\|_{E_{k}}=\|u\|_{H_{0}^{2 m+k}(\Omega)}+\|v\|_{H_{0}^{k}(\Omega)} \leq R_{k}\right\}, \\
\left\|S(t)\left(u_{0}, v_{0}\right)\right\|_{E_{k}}^{2}=\|u\|_{H_{0}^{2 m+k}(\Omega)}^{2}+\|v\|_{H_{0}^{k}(\Omega)}^{2} \leq C_{2}^{\prime}\left(R_{k}\right)+\frac{C_{2}}{\alpha_{2}}=C_{8} .
\end{gathered}
$$

where $t \geq 0, \quad\left(u_{0}, v_{0}\right) \in B$, it indicates $\{S(t)\}(t \geq 0)$ is uniformly bounded in $E_{k}$.

2) According to lemma 2, $\forall\left(u_{0}, v_{0}\right) \in E_{k}$, for $t \geq \max \left(t_{1}, t_{2}\right)$

$$
\left\|S(t)\left(u_{0}, v_{0}\right)\right\|_{E_{k}}^{2}=\|u\|_{H_{0}^{2 m+k}(\Omega)}^{2}+\|v\|_{H_{0}^{k}(\Omega)}^{2} \leq R_{0}^{2}+R_{1}^{2} \leq R_{k}^{2} .
$$

Thus, semigroup $S(t)$ exists bounded absorbing set $B_{0 k}$.

3) $E_{k} \subset E_{0}$ is compact embedded, it indicates the bounded set in $E^{k}$ is the compact set in $E_{0}$, so semigroup operator $S(t)$ is completely continuous. Furthermore we can get semigroup operator $S(t)$ exists a compact family of the

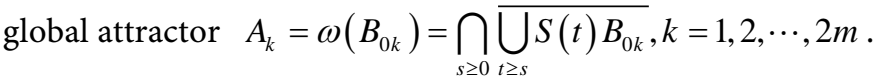

Theorem 3 is proved.

\section{Estimation of the Dimension of the Family of Global Attractors}

Let's consider the linearization problem of (1.1)-(1.3)

$$
\begin{gathered}
U_{t t}+M\left(\left\|\nabla^{m} u\right\|_{p}^{p}\right)(-\Delta)^{2 m} U \\
+p M^{\prime}\left(\left\|\nabla^{m} u\right\|_{p}^{p}\right)\left(\int_{\Omega}\left|\nabla^{m} u\right|^{p-2} \nabla^{m} u \cdot \nabla^{m} U \mathrm{~d} x\right)(-\Delta)^{2 m} u \\
+\beta(-\Delta)^{2 m} U_{t}+g^{\prime}(u) U=0 . \\
U(x, t)=0, \frac{\partial^{i} U}{\partial v^{i}}, k=1,2, \cdots, 2 m-1, t>0 . \\
U(x, 0)=\xi_{1}, U_{t}(x, 0)=\xi_{2} .
\end{gathered}
$$

where $\left(\xi_{1}, \xi_{2}\right) \in E_{k}, \quad\left(u, u_{t}\right)=S(t)\left(u_{0}, u_{1}\right)$ is the solution of the problem (1.1)(1.3) which take $\left(u_{0}, u_{1}\right) \in A_{k}$ as the initial value. $\left(u_{0}, u_{1}\right) \in A, S(t): E_{k} \rightarrow E_{k}$, it can be proved that for any $\left(\xi_{1}, \xi_{2}\right) \in E_{k}$, linearized initial boundary value problem (5.1)-(5.3) have unique solution $\left(U(t), U_{t}(t)\right) \in L^{\infty}\left([0,+\infty) ; E_{k}\right)$.

Theorem 4. The Frechet derivative of mapping $S(t): E_{k} \rightarrow E_{k}$ is the linear operator $T_{k}:\left(\xi_{1}, \xi_{2}\right) \rightarrow\left(U(t), U_{t}(t)\right)$ on $\phi_{0}=\left(u_{0}, u_{1}\right)$, thus for any $t>0, R>0$, mapping $S(t): E_{k} \rightarrow E_{k}$ is Frechet differentiable on $E_{k}$, and $\left(U(t), U_{t}(t)\right)$ is the solution to Equations (5.1)-(5.3). Proof. Let $\phi_{0}=\left(u_{0}, v_{0}\right) \in E_{k}$, $\overline{\phi_{0}}=\left(u_{0}+\xi_{1}, v_{0}+\xi_{2}\right) \in E_{k}$, and $\left\|\bar{\phi}_{0}\right\|_{E_{k}} \leq R$, define $(u, v)=S(t) \phi_{0}$, $(\bar{u}, \bar{v})=S(t) \overline{\phi_{0}}, c$ is a constant. We can get the Lipschitz property of $\mathrm{S}(\mathrm{t})$ on the bounded set $E_{k}$, that is

$$
\left\|S(t) \phi_{0}-S(t) \bar{\phi}_{0}\right\|_{E_{k}}^{2} \leq \mathrm{e}^{c t}\left\|\left(\xi_{1}, \xi_{2}\right)\right\|_{E_{k}}^{2} .
$$

Let $\sigma=\bar{u}-u-U$ is the solution of problem (1.1)-(1.3), we have 


$$
\begin{gathered}
\sigma_{t t}+M\left(\left\|\nabla^{m} u\right\|_{p}^{p}\right)(-\Delta)^{2 m} \sigma+\beta(-\Delta)^{2 m} \sigma_{t}=-h, \\
\sigma(0)=\sigma_{t}(0)=0 .
\end{gathered}
$$

Let $s=\left\|\nabla^{m} u\right\|_{p}^{p}, \bar{s}=\left\|\nabla^{m} \bar{u}\right\|_{p}^{p}$, we obtained

$$
\begin{aligned}
h= & {[M(\bar{s})-M(s)](-\Delta)^{2 m} \bar{u}-M^{\prime}(s) s^{\prime} \nabla^{m} U(-\Delta)^{2 m} u } \\
& -\left(g(u)-g(\bar{u})+g^{\prime}(u) U\right) \\
= & M^{\prime}\left(\left\|\xi_{1}\right\|_{p}^{p}\right)\left(\left\|\xi_{1}\right\|_{p}^{p}\right)^{\prime} \nabla^{m}(\bar{u}-u)(-\Delta)^{2 m} \bar{u} \\
& -M^{\prime}\left(\left\|\nabla^{m} u\right\|_{p}^{p}\right)\left(\left\|\nabla^{m} u\right\|_{p}^{p}\right)^{\prime} \nabla^{m}(\bar{u}-u)(-\Delta)^{2 m} u \\
& +M^{\prime}\left(\left\|\nabla^{m} u\right\|_{p}^{p}\right)\left(\left\|\nabla^{m} u\right\|_{p}^{p}\right)^{\prime} \nabla^{m} \sigma(-\Delta)^{2 m} u-\left(g(u)-g(\bar{u})+g^{\prime}(u) U\right) \\
= & h_{1}+h_{2}+h_{3} .
\end{aligned}
$$

where $\xi_{1}=(1-\alpha) \bar{s}+\alpha s, 0<s<1$.

$$
\begin{gathered}
h_{1}=M^{\prime}\left(\left\|\nabla^{m} u\right\|_{p}^{p}\right)\left(\left\|\nabla^{m} u\right\|_{p}^{p}\right)^{\prime} \nabla^{m} \sigma(-\Delta)^{2 m} u \\
h_{2}=M^{\prime}\left(\left\|\xi_{1}\right\|_{p}^{p}\right)\left(\left\|\xi_{1}\right\|_{p}^{p}\right)^{\prime} \nabla^{m}(\bar{u}-u)(-\Delta)^{2 m} \bar{u} \\
-M^{\prime}\left(\left\|\nabla^{m} u\right\|_{p}^{p}\right)\left(\left\|\nabla^{m} u\right\|_{p}^{p}\right)^{\prime} \nabla^{m}(\bar{u}-u)(-\Delta)^{2 m} u .
\end{gathered}
$$

Let $N^{\prime}\left(\xi_{1}\right)=M^{\prime}\left(\left\|\xi_{1}\right\|_{p}^{p}\right)\left(\left\|\xi_{1}\right\|_{p}^{p}\right)^{\prime}$, then

$$
\begin{aligned}
h_{2} & =\left[\left(N^{\prime}\left(\xi_{1}\right)-N^{\prime}(s)\right)(-\Delta)^{2 m} \bar{u}+N^{\prime}(s)(-\Delta)^{2 m}(\bar{u}-u)\right] \nabla^{m}(\bar{u}-u) \\
& =N^{\prime \prime}\left(\xi_{2}\right)(1-\alpha)\left(\nabla^{m}(\bar{u}-u)\right)^{2}(-\Delta)^{2 m} \bar{u}+N^{\prime}(s)(-\Delta)^{2 m}(\bar{u}-u) \nabla^{m}(\bar{u}-u) .
\end{aligned}
$$

where $\xi_{2}=\theta \xi_{1}+(1-\theta) s, 0<\theta<1$, then

$$
h_{3}=-\left(g(u)-g(\bar{u})+g^{\prime}(u) U\right) .
$$

Take the inner product of (5.5) with $(-\Delta)^{k} \sigma_{t}$, we get

$$
\begin{aligned}
& \frac{1}{2} \frac{\mathrm{d}}{\mathrm{d} t}\left\|\nabla^{k} \sigma_{t}\right\|^{2}+M(s) \frac{1}{2} \frac{\mathrm{d}}{\mathrm{d} t}\left\|\nabla^{2 m+k} \sigma\right\|^{2}+\beta\left\|\nabla^{2 m+k} \sigma_{t}\right\|^{2} \\
& =\left(h,(-\Delta)^{k} \sigma_{t}\right) .
\end{aligned}
$$

And we get

$$
\begin{aligned}
\left|\left(-h,(-\Delta)^{k} \sigma_{t}\right)\right| & =\left|\left(-\left(h_{1}+h_{2}+h_{3}\right),(-\Delta)^{k} \sigma_{t}\right)\right| \\
& \leq\left|\left(h_{1},(-\Delta)^{k} \sigma_{t}\right)\right|+\left|\left(h_{2},(-\Delta)^{k} \sigma_{t}\right)\right|+\left|\left(h_{3},(-\Delta)^{k} \sigma_{t}\right)\right| .
\end{aligned}
$$

Let $w=u-\bar{u}$, according to lemma 1, lemma 2, differential mean value theorem, interpolation inequality and Poincare's inequality, we obtained 


$$
\begin{gathered}
\left|\left(h_{1},(-\Delta)^{k} \sigma_{t}\right)\right|=\left|\left(M^{\prime}(s) s^{\prime}(-\Delta)^{2 m} u \nabla^{k} \sigma,(-\Delta)^{k} \sigma_{t}\right)\right| \\
\leq C_{8}\left\|(-\Delta)^{2 m} u\right\|\left\|\nabla^{m+k} \sigma\right\|\left\|\nabla^{k} \sigma_{t}\right\| \\
\leq \frac{C_{9} \lambda_{1}^{-m}}{2}\left\|\nabla^{2 m+k} \sigma\right\|^{2}+\frac{C_{10}}{2}\left\|\nabla^{k} \sigma_{t}\right\|^{2} . \\
\left|\left(h_{2},(-\Delta)^{k} \sigma_{t}\right)\right|=\mid\left(N^{\prime \prime}\left(\xi_{2}\right)(1-\alpha)(-\Delta)^{2 m} \bar{u}\left(\nabla^{m}(\bar{u}-u)\right)^{2}\right. \\
\left.+N^{\prime}(s)(-\Delta)^{2 m}(\bar{u}-u) \nabla^{m}(\bar{u}-u),(-\Delta)^{k} \sigma_{t}\right) \mid \\
\leq\left\|N^{\prime \prime}\left(\xi_{2}\right)\right\|\left\|_{\infty}\right\|(-\Delta)^{2 m} \bar{u}\left\|_{\infty}\right\| \nabla^{m} w\left\|^{2}\right\| \nabla^{2 k} \sigma_{t} \| \\
+\left\|N^{\prime}(s)\right\| \|_{\infty}\left|\int_{\Omega}(-\Delta)^{2 m}(\bar{u}-u) \nabla^{m}(\bar{u}-u)(-\Delta)^{k} \sigma_{t} \mathrm{~d} x\right| \\
\leq C_{11}\left\|\nabla^{2 k} \sigma_{t}\right\|\left\|\nabla^{2 m+k} w\right\|^{2}\|w\|^{2}+C_{12}\left\|\nabla^{2 k} \sigma_{t}\right\|\left\|\nabla^{2 m+k} w\right\|^{2}\|w\|^{2} \\
\leq C_{13}\left\|\nabla^{2 k} \sigma_{t}\right\|\left\|\nabla^{2 m+k} w\right\|^{2}+C_{14}\left\|\nabla^{2 k} \sigma_{t}\right\|\left\|\nabla^{2 m+k} w\right\|^{2} \\
\leq \frac{C_{13}^{2}+C_{14}^{2} \lambda_{1}^{-k}\left\|\nabla^{k} \sigma_{t}\right\|^{2}+\frac{C_{15}^{2}+C_{16}^{2}}{2}\left\|\nabla^{2 m+k} w\right\|^{4} .}{2} \\
\leq\left(h_{3},(-\Delta)^{k} \sigma_{t}\right) \mid=\left(-\left(g(u)-g(\bar{u})+g^{\prime}(u) U\right),(-\Delta)^{k} \sigma_{t}\right) \\
=\left(-\left(g^{\prime}(\xi) w,(-\Delta)^{k} \sigma_{t}\right)-\left(g^{\prime}(u) w,(-\Delta)^{k} \sigma_{t}\right)-\left(g^{\prime}(u) \sigma,(-\Delta)^{k} \sigma_{t}\right)\right. \\
\leq(\alpha-1)\left(g^{\prime \prime}(\eta)|w|^{2},(-\Delta)^{k} \sigma_{t}\right)-\left(g^{\prime}(u) \sigma,(-\Delta)^{k} \sigma_{t}\right) \\
\leq C_{17}\left(\left\|\nabla^{2 m+k} w\right\|^{2}+\left\|\nabla^{k} \sigma_{t}\right\|\right)\left\|\nabla^{2 m+k} \sigma_{t}\right\| \\
\leq C_{18}\left(\left\|\nabla^{2 m+k} w\right\|^{4}+\left\|\nabla^{k} \sigma_{t}\right\|^{2}\right)+\frac{\beta}{2}\left\|\nabla^{2 m+k} \sigma_{t}\right\|^{2} .
\end{gathered}
$$

Sum up (5.14)-(5.16), we get

$$
\left(h,(-\Delta)^{k} \sigma_{t}\right) \leq C_{19}\left(\left\|\nabla^{2 m+k} w\right\|^{4}+\left\|\nabla^{2 m+k} \sigma\right\|^{2}+\left\|\nabla^{k} \sigma_{t}\right\|^{2}\right)+\beta\left\|\nabla^{2 m+k} \sigma_{t}\right\|^{2} .
$$

Then substitute inequality (5.14)-(5.17) to inequality (5.12), and by using Young's inequality, Poincare's inequality can obtained

$$
\frac{\mathrm{d}}{\mathrm{d} t}\left[\left\|\nabla^{k} \sigma_{t}\right\|^{2}+\mu\left\|\nabla^{2 m+k} \sigma\right\|^{2}\right] \leq C_{19}\left(\left\|\nabla^{k} \sigma_{t}\right\|^{2}+\mu\left\|\nabla^{2 m+k} \sigma\right\|^{2}+\left\|\nabla^{2 m+k} w\right\|^{4}\right) .
$$

According to Gronwall's inequality, we get

$$
\left\|\nabla^{k} \sigma_{t}\right\|^{2}+\mu\left\|\nabla^{2 m+k} \sigma\right\|^{2} \leq C_{20} \mathrm{e}^{C_{19} t}\left\|\left(\xi_{1}, \xi_{2}\right)\right\|_{E_{k}}^{4} .
$$

According to (5.19), we can get $\left\|\left(\xi_{1}, \xi_{2}\right)^{\mathrm{T}}\right\|_{E_{k}}^{2} \rightarrow 0$, the following is established

$$
\frac{\|\bar{\phi}(t)-\phi(t)-U(t)\|_{E_{k}}^{2}}{\left\|\left(\xi_{1}, \xi_{2}\right)^{\mathrm{T}}\right\|_{E_{k}}^{2}} \leq C_{20} \mathrm{e}^{C_{19} t}\left\|\left(\xi_{1}, \xi_{2}\right)\right\|_{E_{k}}^{2} \rightarrow 0 .
$$

The theorem 4 is proved.

The following will show that the family of the global attractor $A_{k}, k=1,2, \cdots, 2 m$ 
have finite Hausdorff dimension and Fractal dimension.

Theorem 5 In theorem 3, the family of the global attractor $A_{k}$ of equation (1.1)-(1.3) have finite Hausdorff dimension and Fractal dimension, and $d_{H}\left(A_{k}\right)<\frac{1}{6} n, d_{F}\left(A_{k}\right)<\frac{7}{6} n$.

Proof we can write Equation (1.1) as

$$
u_{t t}+M\left(\left\|\nabla^{m} u\right\|_{p}^{p}\right) \nabla^{4 m} u+\beta \nabla^{4 m} u_{t}+g(u)=f(x) .
$$

Suppose $\psi=R_{\varepsilon} \phi=(u, v)^{\mathrm{T}}, \phi=\left(u, u_{t}\right), v=u_{t}+\varepsilon u$, $R_{\varepsilon}:\left(u, u_{t}\right)^{\mathrm{T}} \rightarrow\left(u, u_{t}+\varepsilon u\right)^{\mathrm{T}}$, is a isomorphic mapping, and Equation (5.21) can write as

$$
\psi_{t}+\bar{\Lambda}_{\varepsilon} \psi=\bar{f}
$$

where $\psi=\left\{u, u_{t}+\varepsilon u\right\}^{\mathrm{T}}, \bar{f}=\{0, f(x)\}^{\mathrm{T}}$.

$$
\begin{array}{cc}
\bar{\Lambda}_{\varepsilon}=\left(\begin{array}{cc}
\varepsilon I & -I \\
\left(M\left(\left\|\nabla^{m} u\right\|_{p}^{p}\right)-\beta \varepsilon\right) \nabla^{4 m}+\varepsilon^{2} I+g(u) & \beta \nabla^{4 m}-\varepsilon I
\end{array}\right) \\
\psi_{t}:=F^{\prime}(\psi)=\bar{f}-\bar{\Lambda}_{\varepsilon} \psi . \\
P_{t}=F_{t}(\psi) .
\end{array}
$$

Assume $F: E_{k} \rightarrow E_{k}$ is Frechet differential, then linearize Equation (5.23), we get

$$
P_{t}+\Lambda_{\varepsilon} P=0
$$

where

$$
\begin{aligned}
& \Lambda_{\varepsilon}=\left(\begin{array}{cc}
\varepsilon I & -I \\
\left(M\left(\left\|\nabla^{m} u\right\|_{p}^{p}\right)-\beta \varepsilon\right) \nabla^{4 m}+\varepsilon^{2} I+D \cdot+g^{\prime}(u) \cdot U & \beta \nabla^{4 m}-\varepsilon I
\end{array}\right) \\
& D \cdot U=p M^{\prime}\left(\left\|\nabla^{m} u\right\|_{p}^{p}\right) \int_{\Omega}\left|\nabla^{m} u\right|^{p-2} \nabla^{m} u \cdot \nabla^{m} U \mathrm{~d} x \nabla^{4 m} u, \text { and } P=\left(U, U_{t}+\varepsilon U\right)^{\mathrm{T}}, U
\end{aligned}
$$
is the solution of Equation (5.22).

For a fixed $\left(u_{0}, v_{0}\right) \in E_{k}$, assume $\xi_{1}, \xi_{2}, \cdots, \xi_{n}$ are elements of $E_{k}$, and suppose $U_{1}(t), U_{2}(t), \cdots, U_{n}(t)$ are solutions of linear Equation (5.1), and corresponding initial values are $U_{1}(0)=\xi_{1}, U_{2}(0)=\xi_{2}, \cdots, U_{n}(0)=\xi_{n}$, so we have

$$
\begin{aligned}
& \left\|U_{1}(t) \Lambda U_{2}(t) \Lambda \cdots U_{n}(t)\right\|_{\Lambda E_{k}}^{2} \\
& =\left\|\xi_{1} \Lambda \xi_{2} \Lambda \cdots \xi_{n}\right\|_{\Lambda E_{k}}^{2} \exp \left(\int_{0}^{t} t r F^{\prime}(\psi(\tau)) \cdot Q_{n}(\tau) \mathrm{d} \tau\right) .
\end{aligned}
$$

where $\Lambda$ denotes the exterior product, tr denotes the trace of the operator, $Q_{n}(\tau)$ is the orthogonal projection from space $E_{k}$ to $\operatorname{span}\left\{U_{1}(t), U_{2}(t), \cdots, U_{n}(t)\right\}$.

For a given time $\tau$, assume $\omega_{j}(\tau)=\left(\zeta_{j}(\tau), \eta_{j}(\tau)\right)^{\mathrm{T}},(j=1,2, \cdots, n)$ is orthonormal basis of $\operatorname{span}\left\{U_{1}(t), U_{2}(t), \cdots, U_{n}(t)\right\}$.

Define the inner product of $E_{k}$ 


$$
((\zeta, \eta),(\bar{\zeta}, \bar{\eta}))=\left(\nabla^{2 m+k} \zeta, \nabla^{2 m+k} \bar{\zeta}\right)+\left(\nabla^{k} \eta, \nabla^{k} \bar{\eta}\right)
$$

To sum up, we get

$$
\begin{aligned}
\operatorname{trF}_{t}(\psi(\tau)) Q_{n}(\tau) & =\sum_{j=1}^{n}\left(F_{t}(\psi(\tau)) Q_{n}(\tau) \omega_{j}(\tau), \omega_{j}(\tau)\right)_{E_{k}} \\
& =\sum_{j=1}^{n}\left(F_{t}(\psi(\tau)) \omega_{j}(\tau), \omega_{j}(\tau)\right)_{E_{k}} .
\end{aligned}
$$

And

$$
\left(F_{t}(\psi(\tau)) \omega_{j}(\tau), \omega_{j}(\tau)\right)_{E_{k}}=-\left(\Lambda_{\varepsilon} \omega_{j}, \omega_{j}\right) .
$$

By using Holder inequality, Young's inequality and Poincare inequality

$$
\begin{aligned}
\left(\Lambda_{\varepsilon} \omega_{j}, \omega_{j}\right)= & \left(\left(\varepsilon \zeta_{j}-\eta_{j},\left(M\left(\left\|\nabla^{m} u\right\|_{p}^{p}\right)-\beta \varepsilon\right) \nabla^{4 m} \zeta_{j}+\varepsilon^{2} \zeta_{j}+D \cdot \zeta_{j}\right.\right. \\
& \left.\left.+g^{\prime}(u) \cdot \zeta_{j}+\beta \nabla^{4 m} \eta_{j}-\varepsilon \eta_{j}\right),\left(\zeta_{j}, \eta_{j}\right)\right) \\
= & \varepsilon\left\|\nabla^{2 m+k} \zeta_{j}\right\|^{2}+\left(M\left(\left\|\nabla^{m} u\right\|_{p}^{p}\right)-\beta \varepsilon-1\right)\left(\nabla^{2 m+k} \zeta_{j}, \nabla^{2 m+k} \eta_{j}\right) \\
& +\varepsilon^{2}\left(\nabla^{k} \zeta_{j}, \nabla^{k} \eta_{j}\right)+\left(\nabla^{k} \cdot \nabla \zeta_{j}, \nabla^{k} \eta_{j}\right)+\left(\nabla^{k}\left(g^{\prime}(u) \cdot \zeta_{j}\right), \nabla^{k} \eta_{j}\right) \\
& +\beta\left\|\nabla^{2 m+k} \eta_{j}\right\|^{2}-\varepsilon\left\|\nabla^{k} \eta_{j}\right\|^{2} \\
\geq & \gamma_{1}\left\|\nabla^{2 m+k} \zeta_{j}\right\|^{2}+\gamma_{2}\left\|\nabla^{k} \eta_{j}\right\|^{2}-C_{23}\left\|\nabla^{k} \zeta_{j}\right\|^{2} .
\end{aligned}
$$

where

$$
\begin{aligned}
& \gamma_{1}=\frac{1}{2}\left(3 \varepsilon+C_{21} \lambda_{j}^{-m-k}-\beta \varepsilon-\varepsilon^{2} \lambda_{j}^{-2 m}-C_{22} \lambda_{j}^{-2 m-k}\right), \\
& \gamma_{2}=\frac{1}{2}\left((2 \beta+\varepsilon-\beta \varepsilon) \lambda_{j}^{-2 m}+C_{21} \lambda_{j}^{2 m}-\frac{C_{23}}{2}-\varepsilon-\varepsilon^{2}-C_{22}\right) .
\end{aligned}
$$

Let $\bar{\delta}=\min \left\{\gamma_{1}, \gamma_{2}\right\}, \delta=\min \left\{\bar{\delta} \lambda_{j}^{2 m+k}, \bar{\delta} \lambda_{j}^{k}\right\}$, then we get

$$
\begin{aligned}
& \left(F_{t}(\psi(\tau)) \omega_{j}(\tau), \omega_{j}(\tau)\right)_{E_{k}} \\
& =-\left(\Lambda_{\varepsilon} \omega_{j}, \omega_{j}\right) \leq-\bar{\delta}\left(\left\|\nabla^{2 m+k} \zeta_{j}\right\|^{2}+\left\|\nabla^{k} \eta_{j}\right\|^{2}\right)+C_{23}\left\|\nabla^{k} \zeta_{j}\right\|^{2} \\
& \leq \delta\left(\left\|\zeta_{j}\right\|^{2}+\left\|\eta_{j}\right\|^{2}\right)+C_{23}\left\|\nabla^{k} \zeta_{j}\right\|^{2} .
\end{aligned}
$$

Because of $\omega_{j}(\tau)=\left(\zeta_{j}(\tau), \eta_{j}(\tau)\right)^{\mathrm{T}},(j=1,2, \cdots, n)$ is orthonormal basis, thus

$$
\begin{gathered}
\left\|\zeta_{j}\right\|^{2}+\left\|\eta_{j}\right\|^{2}=1 . \\
\sum_{j=1}^{N}\left(F_{t}(\psi(\tau)) \omega_{j}(\tau), \omega_{j}(\tau)\right)_{E_{k}} \leq-N \delta+C_{23} \sum_{j=1}^{N}\left\|\nabla^{k} \zeta_{j}\right\|^{2} .
\end{gathered}
$$

There exists $s=\frac{k}{2 m+k}$, and $0 \leq s<1$, we have

$$
\sum_{j=1}^{N}\left\|\nabla^{k} \zeta_{j}\right\|^{2} \leq \sum_{j=1}^{N} \lambda_{j}^{s-1} .
$$


So

$$
\operatorname{tr} F_{t}(\psi(\tau)) \cdot Q_{n}(\tau) \leq-N \delta+C_{23} \sum_{j=1}^{N} \lambda_{j}^{s-1}
$$

Then assume $\left(u_{0}, u_{1}\right) \in A, \mathrm{~A}$ is bounded absorbing set of $E_{k}$,

$$
\begin{gathered}
\psi(t)=\left(u(t), u_{t}(t)+\varepsilon u(t)\right) \in D(A), D(A)=\left\{u \in H^{2 m}, A u \in L^{2}(\Omega)\right\}, \text { let } \\
q_{N}(t)=\sup _{\substack{\psi_{0} \in A \\
\eta_{j} \in E_{k} \\
\left\|\nabla^{k} \eta_{j}\right\| \leq 1}} \sup _{\substack{t \\
t}}\left(t F_{t}\left(S(\tau) \psi_{0}\right) \cdot Q_{n}(\tau) \mathrm{d} \tau\right) . \\
q_{N}=\lim _{t \rightarrow \infty} q_{N}(t) .
\end{gathered}
$$

By the inequality (5.34)-(5.36), we can get

$$
q_{N} \leq-N \delta+C_{23} \sum_{j=1}^{N} \lambda_{j}^{s-1} .
$$

Thus, the Lyapunov exponent of $B_{0 k}$ is uniformly bounded, that is

$$
\sigma_{1}+\sigma_{2}+\cdots+\sigma_{n} \leq-N \delta+C_{23} \sum_{j=1}^{N} \lambda_{j}^{s-1} .
$$

So, there exists a $s \in[0,1)$, such that

$$
\left(q_{j}\right)_{+} \leq-N \delta+C_{23} \sum_{j=1}^{N} \lambda_{j}^{s-1} \leq C_{23} \sum_{j=1}^{N} \lambda_{j}^{s-1} \leq \frac{N \delta}{7} .
$$

where $\lambda_{j}$ is the eigenvalue of $A$, and $\lambda_{1}<\lambda_{2}<\cdots<\lambda_{m}$, then

$$
q_{N} \leq-N \delta\left(1-\frac{C_{23}}{N \delta} \sum_{j=1}^{N} \lambda_{j}^{s-1}\right) \leq-\frac{6}{7} N \delta .
$$

So

$$
\max _{1 \leq j \leq n} \frac{\left(q_{j}\right)_{+}}{\left|q_{N}\right|} \leq \frac{1}{6} .
$$

Thus, we can get the conclusion $d_{H}\left(A_{k}\right)<\frac{1}{6} n, d_{F}\left(A_{k}\right)<\frac{7}{6} n$.

\section{Conflicts of Interest}

The authors declare no conflicts of interest regarding the publication of this paper.

\section{References}

[1] Kirchhoff, G. (1883) Vorlesungen Fiber Mechanic. Tenbner, Stuttgart.

[2] Masamro, H. and Yoshio, Y. (1991) On Some Nonlinear Wave Equations 2: Global Existence and Energy Decay of Solutions. Journal of the Faculty of Science, University of Tokyo, Section, 38, 239-250.

[3] Cavalcanti, M.M., Cavalcanti, V.N.D., Filho, J.S.P. and Soriano, J.A. (1998) Existence and Exponential Decay for a Kirchhoff-Carrier Model with Viscosity. Journal of Mathematical Analysis and Applications, 226, 20-40.

https://doi.org/10.1006/jmaa.1998.6057 
[4] Ono, K. (1997) On Global Existence, Decay, and Blow Up of Solutions for Some Mildly Degenerate Nonlinear Kirchhoff Strings. Journal of Differential Equations, 137, 173-301. https://doi.org/10.1006/jdeq.1997.3263

[5] Ono, K. (1997) On Global Existence, Asymptotic Stability and Blowing Up of Solutions for Some Degenerate Non-Linear Wave Equations of Kirchhoff Type with a Strong Dissipation. Mathematical Methods in the Applied Sciences, 20, 151-177. https://doi.org/10.1002/(SICI)1099-1476(19970125)20:2<151::AID-MMA851>3.0.C $\underline{\mathrm{O} ; 2-0}$

[6] Yang, Z. and Li, X. (2011) Finite Dimensional Attractors for the Kirchhoff Equation with a Strong Dissipation. Journal of Mathematical Analysis and Applications, 375, 579-593. https://doi.org/10.1016/j.jmaa.2010.09.051

[7] Lin, G.G. and Xu, G.G. (2011) Global Attractors and Their Dimension Estimation for the Generalized Boussinesq Equation. China Science and Technology information, 5, 54-55.

[8] Lin, G.G. (2019) Dynamic Properties of Several Kinds of the K Equations. Chongqing University Press, Chongqing.

[9] Wang, M.X., Tian, C.C. and Lin, G.G. (2014) Global Attractor and Dimension Estimation for a 2D Generalized Anisotropy Kuramoto-Sivashinsky Equation. International Journal of Modern Nonlinear Theory and Application, 3, 163-172. https://doi.org/10.4236/ijmnta.2014.34018

[10] Lin, G.G. and Gao, Y.L. (2017) The Global and Exponential Attractor for the Higher-Order Kirchhoff-Type Equation with Strong Linear Damping. Journal of Mathematics Research, 9, 145-167. https://doi.org/10.5539/jmr.v9n4p145

[11] Lin, G.G. and Guan, L.P. (2019) The Family of Global Attractor and Their Dimension Estimates for Strongly Damped High-Order Kirchhoff Equation. Acta Analysis Functionalis Applicata, 21, 268-281.

[12] Lin, G.G., Xia, F.F. and Xu, G.G. (2013) The Global and Pullback Attractors for a Strongly Damped Wave Equation with Delay. International Journal of modern Nonlinear Theory and Application, 2, 209-218. https://doi.org/10.4236/ijmnta.2013.24029

[13] Nakao, M. (2009) An Attractor for a Nonlinear Dissipative Wave Equation of Kirchhoff Type. Journal of Mathematical Analysis and Applications, 353, 652-659. https://doi.org/10.1016/j.jmaa.2008.09.010

[14] Sun, Y.T., Gao, Y.L., Lin, G.G. (2016) The Global Attractors for the Higher-Order Kirchhoff-Type Equation with Nonlinear Strongly Damped Term. International Journal of Modern Nonlinear Theory and Application, 5, 203-217.

https://doi.org/10.4236/ijmnta.2016.54019

[15] Chen, L., Wang, W. and Lin, G.G. (2016) The Global Attractors and Their Hausdorff and Fractal Dimensions Estimation for the Higher-Order Nonlinear Kirchhoff-Type Equation with Strong Linear Damping. Journal of Advances in Mathematics, 5,185-202. https://doi.org/10.4236/ijmnta.2016.54018

[16] Lin, G.G. (2011) Nonlinear Evolution Equation. Yunnan University Press, Kunming. 